\title{
Prediction of performance of Stirling engine using least squares support machine technique
}

\author{
Mohammad H. Ahmadi ${ }^{1, a}$, Mohammad Ali Ahmadi ${ }^{2}$, Milad Ashouri ${ }^{1}$, F. Razie \\ Astaraei $^{1}$, R. Ghasempour ${ }^{3}$ and Fethi Aloui ${ }^{3}$ \\ 1 Renewable Energies and Environmental Department, Faculty of New Sciences and Technologies, University of Tehran, \\ Tehran, Iran \\ 2 Department of Petroleum Engineering, Ahwaz Faculty of Petroleum Engineering, Petroleum University of Technology (PUT), \\ Ahwaz, Iran \\ 3 University of Valenciennes, LAMIH CNRS UMR 8201, Department of Mechanics, Campus Mont Houy, 59313 Valenciennes \\ Cedex 9, France
}

Received 2 July 2015, Accepted 22 October 2015

\begin{abstract}
Stirling engine is an environmental friendly heat engine which could reduce $\mathrm{CO}_{2}$ emission through combustion process. Output power, shaft torque and brake specific fuel consumption represent the efficiency and robustness of the Stirling engines. The present research tries to determine the three aforementioned parameters with high accuracy and low uncertainty. In this research a new type of intelligent models named "least square support vector machine (LSSVM) was employed to predict output power, shaft torque and brake specific fuel consumption. Furthermore, high accurate actual values of the required parameters from previous studies were implemented to develop the robust intelligent model. A great advantage of LSSVM model over ANN is that in the present model over fitting does not happen. Expected statistical parameters of the suggested intelligent model have been indicated and validate the high efficiency of the suggested LSSVM model. Good agreement between LSSVM results and actual values was observed. Solutions obtained from the developed support vector machine model could help us in exact designing of Stirling engine with low uncertainty.
\end{abstract}

Key words: Support vector machine / leverage approach / stirling engine / torque / power

\section{Introduction}

Recent advances in alternative and clean energy systems have helped us to encounter the crises of fossil fuel reductions in the world. Therefore, many researchers focused on new energy conversion devices which can use various sources like bio-mass, solar, waste heat etc. in an efficient and environmentally friendly manner. Among these, Stirling engine is a promising technology which is able to generate power with hight thermal efficiency. This is an external combustion engine operating in a wide temperature difference and good combustion control. The Stirling engine works by compression and expansion of different working fluids such as air, hydrogen and helium in two separated spaces. Two pistons with phase angle transfer the working fluid between these spaces $[1,2]$. The thermophysical properties of the working fluids such as high

\footnotetext{
${ }^{a}$ Corresponding author:

mohammadhosein.ahmadi@gmail.com
}

heat capacity, high thermal conductivity and low viscosity, heat transfer coefficient, temperature difference between cold and hot ends, regenerator efficiency, mechanical connections, charge pressure and impermeability ratio play an important role in the performance of the system [3]. In recent years many studies, theoretically and experimentally, have been done to improve the performance of the Stirling engines. The first modern engine was developed by Philips Company in 1937. They produced a number of Stirling engines up to $336 \mathrm{KWe}$ [4]. Prodesser developed a Stirling engine working with the stack gas of a biomass furnace in country zones [5]. It generates $3.2 \mathrm{~kW}$ power output at $600 \mathrm{rpm}$ under 33 bars charge pressure.

Sripakagorn and Srikam [6] established a beta-type operating in moderate temperature ranges. The engine produces $95.4 \mathrm{~W}$ at $360 \mathrm{rpm}, 773 \mathrm{~K}$ hot source temperature and 7 bars mean pressure. Karabulut et al. [7] investigated a Stirling engine experimentally with a lever-controlled displacer driving mechanism with helium as the working 


\section{Nomenclature}

\begin{tabular}{|ll|}
\hline AARD & Average absolute relative deviation \\
ARD & Average relative deviation \\
ANNs & Artificial neural networks \\
& Intercept of the liner regression \\
& in the modified SVM procedure \\
GA & Genetic algorithm \\
$H$ & Hat matrix \\
LSSVM & Least square support vector machine \\
MSE & Mean square error \\
$N$ & Number of data points \\
RMSE & Root mean square error \\
SVM & Support vector machine \\
$e_{k}$ & Regression error \\
$\gamma$ & Regularization parameter \\
$\alpha_{k}$ & Lagrange multipliers \\
$\sigma^{2}$ & RBF kernel parameter in the LSSVM model \\
$w$ & Weight vector \\
$x$ & Input vector of parameters of the model \\
$y$ & Connotes the outputs \\
\hline
\end{tabular}

fluid. The maximum torque and power are $3.99 \mathrm{Nm}$ and $183 \mathrm{~W}$ at 4 bars charge pressure and $533 \mathrm{~K}$ hot-end temperature. Cheng and $\mathrm{Yu}$ [8] proposed a numerical approach for a beta-type rhombic-drive Stirling engine. Effectiveness of the regenerative channel, non-isothermal effects and the thermal resistance of the heating head were considered in the model. Also, the geometrical parameters were introduced into the model. Several operational and geometrical variables were evaluated through a numerical model developed by Chen et al. [9] based on construction of a c-type twin-power piston Stirling engine. Some improvements were done in the numerical model. The results showed the importance of regenerator in thermal efficiency where the engine speed affected the engine power. Formosa and Despesse [10] developed a model which consisted the imperfect regeneration, the dead volume, and the external and internal thermal transfer effects on engine performance. The model also included the effectiveness of the heat exchangers and the critical flaws of the regenerator.

More recently, various predicting strategies including fuzzy logic, ANN, genetic algorithms, finite element models and the Taguchi method have been introduced to predict the performance of the system. These measures, if used well, help to avoid the time-consuming process of experimental investigations and reduce the cost problems of research studies. These methods consist of time series such as the auto-regressive moving average (ARMA), the auto-regressive conditional heteroskedasticity $(\mathrm{ARCH})$ models $[11,12]$, the linear regression model [13], the grey theory model [14,15], the support vector machine (SVM) [16, 17], adaptive fuzzy logic algorithms [18,19] and artificial neural networks (ANNs) [20,21], or other methods [22].

ANN is a method being used in different applications including manufacturing, signal processing and optimization of energy systems [23]. ANN is based on neural networks of the brain and is used to solve nonlinear problems and complex models. The literature review reveals that no application of ANN has been used on Stirling engine. Although its application on other heat engines has shown good results [24].

The ANN method has been widely used to predict the performance of various problems [25-36]. Although, during optimizing of the model parameters, there might be a random initialization of the networks and ceasing criteria change [36-40]. These conditions may prevent a satisfactory result of ANN application on external predictions indicating external inputs excluding the employed optimization and test methods of corresponding networks.

$\mathrm{SVM}$ is an effective procedure evolved from the machine-learning community [37-42]. A SVM is a device for number of related supervised learning ways that analyze data and identify patterns, applied for regression analysis. The SVM is assumed as a non-probabilistic binary linear classifier [37-42].

According to the particular formulation of the SVM method, a few solutions can be detected and either linear or nonlinear regressions can be followed for solving the corresponding issues [37-42]. LSSVM is commonly applied in oil systems [38, 43-45]. The objective is to propose math-based nonlinear relationships between the available experimental information considered as inputs of the models (Rotation speed, temperature of heat source, Pressure, Fuel) and the desired outputs (Brake Specific fuel consumption, Torque and power). For this purpose, appropriate mathematical tools are required.

The present paper highlights the use of LSSVM method for building new predictive tool for estimating efficiency variables in Stirling engines with the help of Stirling engine experimental outcomes for the first time $[46,47]$.

\section{Model development}

One of the most well-known mathematical methods to erect commensurate relations between the factors of a definite mathematical issue is the SVM which augments the probability of achieving the global optima of a quandary and reduces the chance of over-or underfitting issues of non-linear regression ways [37, 38, 4345, 48-51]. Swifter SVM procedure is evolved by Suykens and Vandewalle [38] concentrating on solving a number of linear equations instead of a twisted set of nonlinear ones in the corresponding SVM calculation steps. A point to consider is that the improved SVM procedure (LSSVM) [38], profits from the advantages of the original version of the algorithm as well. The regression error of the LSSVM [38] approach is identified as the difference between the estimated and forecasted property values and measured ones [46,47], which is noticed as an extension to the limit of the optimization quandary. In traditional SVM procedure, the value of the regression error is commonly optimized during the determinations while 
in the LSSVM [38], the error is mathematically determined $[37,38,43-45,48-51]$. The cost function (penalized cost function) of the employed strategy has been characterized as below [37, 38, 43-45, 48-51]:

$$
Q_{L S S V M}=\frac{1}{2} w^{T} w+\gamma \sum_{k=1}^{N} e_{k}^{2}
$$

Subject to the following limit $[38,43-45]$ :

$$
y_{k}=w^{T} \phi\left(x_{k}\right)+b+e_{k} \quad k=1,2, \ldots, N
$$

In which $x$ stands for the input vector of parameters of the model, $y$ connotes the outputs, $b$ represents the intercept of the liner regression in the modified SVM procedure, $w$ is the regression weight, $e_{k}$ implied by the regression error for $N$ training objects, $\gamma$ represents the relative weight of the summation of the regression errors compared to the regression weight (first right hand side of Eq. (1)), $\phi$ is the feature map, mapping the possible area to a high dimensional feature space, where the measured data can be linearly separable by a hyper plane determined by the pair $\left(w \in R^{m}, b \in R\right)$, and superscript $T$ describes the transpose matrix. The weight coefficient $(w)$ is commonly written as follows $[37,38,43-45,48]$ :

$$
w=\sum_{k=1}^{N} \alpha_{k} x_{k}
$$

where $[37,38,43-45,48-51]$,

$$
\alpha_{k}=2 \gamma e_{k}
$$

Therefore, Equation (2) can be penned as follows [37,38, $43-45,48-51]$ :

$$
y=\sum_{k=1}^{N} \alpha_{k} x_{k}^{T} x+b
$$

Thus, the Lagrange multipliers $\left(\alpha_{k}\right)$ are indicated as [37, $38,43-45,48-51]$ :

$$
\alpha_{k}=\frac{\left(y_{k}-b\right)}{x_{k}^{T} x+(2 \gamma)^{-1}}
$$

Equation (7) can be utilized as a further nonlinear regression employing the Kernel function as follows $[37,38,43-45,48-51]$ :

$$
f(x)=\sum_{k=1}^{N} \alpha_{k} K\left(x, x_{k}\right)+b
$$

where $K\left(x x_{k}\right)$ stands for the Kernel function based on the inner product of the two vectors $x$ and $x_{k}$ in the possible denoted by the inner product of the vectors $\Phi(x)$ and $\Phi\left(x_{k}\right)$ as follows $[37,38,43-45,48-51]$ :

$$
K\left(x, x_{k}\right)=\Phi(x)^{T} \Phi\left(x_{k}\right)
$$

where the radial principle function (RBF) Kernel has been utilized as follows [37, 38, 43-45, 48-51]:

$$
K\left(x, x_{k}\right)=\exp \left(-\left\|x_{k}-x\right\|^{2} / \sigma^{2}\right)
$$

In which $\sigma$ stands for a decision parameter, which is achieved by an outside optimization procedure. The mean square error (MSE) of the outcomes of the final model can be specified as follows [38]:

$$
M S E=\frac{\sum_{i=1}^{n}\left(P_{\text {rep. } / \text { pred }, \mathrm{i}}-P_{\mathrm{exp}, i}\right)^{2}}{n s}
$$

In which subscripts rep./pred. and exp denote the estimated, and measured output (Brake specific fuel consumption, power and torque) values, $[46,47]$ respectively, and $n s$ is the number of samples from the primary population. In the present investigation, the LSSVM [38] algorithm is employed.

\section{Leverage approach}

Outlier detection is very prominent in evolving the mathematical models. In fact, outlier detection is to recognize individual datum which may vary from the bulk of the data present in a dataset [52]. The corresponding ways commonly comprise of numerical and graphical procedures [52-54]. The Leverage approach [52-54] is deemed to be a confident procedure for outlier detection that copes with the values of the residuals and a matrix identified as Hat matrix comprised of the measured data and the estimated values accomplished from a model. The main usage criterion of the procedure is to utilize a LSSVM method, which is able of adequate calculation/estimation of the data of interest. The Leverage or Hat indices are calculated based on Hat matrix $(H)$ with the following definition [52-54]:

$$
H=X\left(X^{T} X\right)^{-1} X^{T}
$$

where $X$ is a $(n \times k)$ matrix, in which $n$ indicates the data (rows) and $k$ represents the parameters of the model (columns), and $T$ shows the transpose matrix. The Hat values of the data in the possible region of the problem are the diagonal elements of the $H$ value.

Having evaluated the $H$ values with Equation (11), the Williams plot is drawn for graphical recognition of the suspended data or outliers. This plot illustrates the correlation of Hat indices and standardized cross-validated residuals $(R)$, which are determined as the distinction between the represented/predicted values and the implemented data. A warning Leverage $\left(H^{*}\right)$ is commonly fixed at the value equal to $3 n / p$, where $n$ is the number of training points and $\mathrm{p}$ is the number of model parameters plus one. The Leverage of three is commonly deemed as a "cut-off" value to comply the points within 73 range (two horizontal redlines) standard deviations from the mean (to cover $99 \%$ commonly distributed data). Existence of 


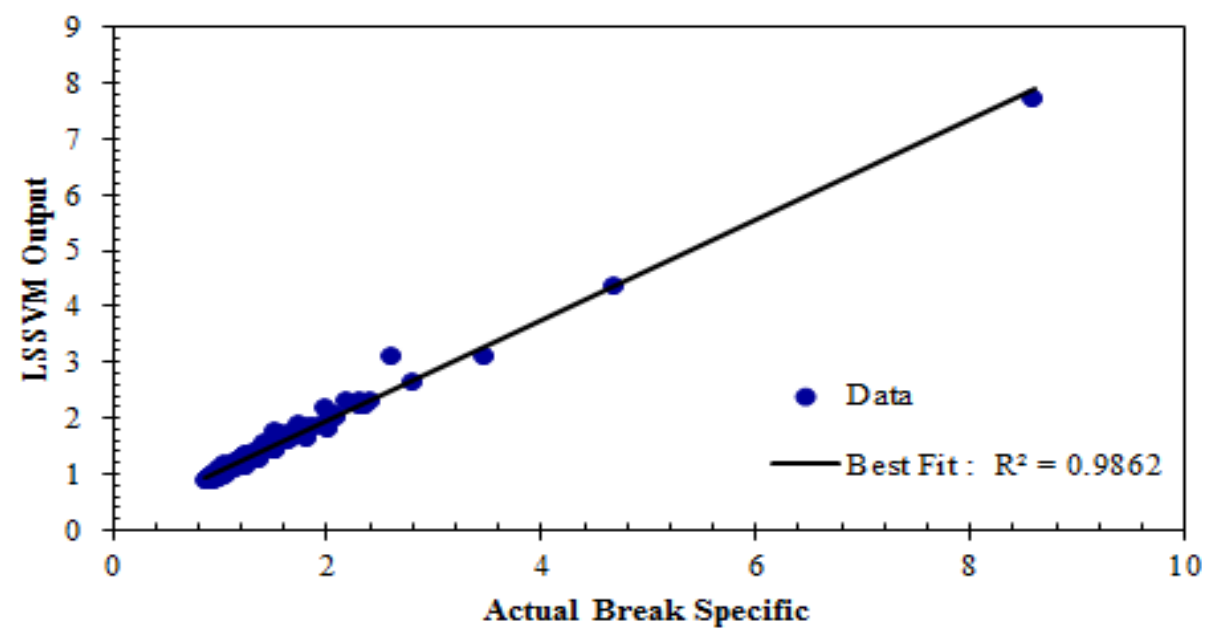

Fig. 1. Linear regression fit between the power estimated by LSSVM method and Brake specific fuel consumption measured in experimental conditions $[46,47]$ for the studied systems.

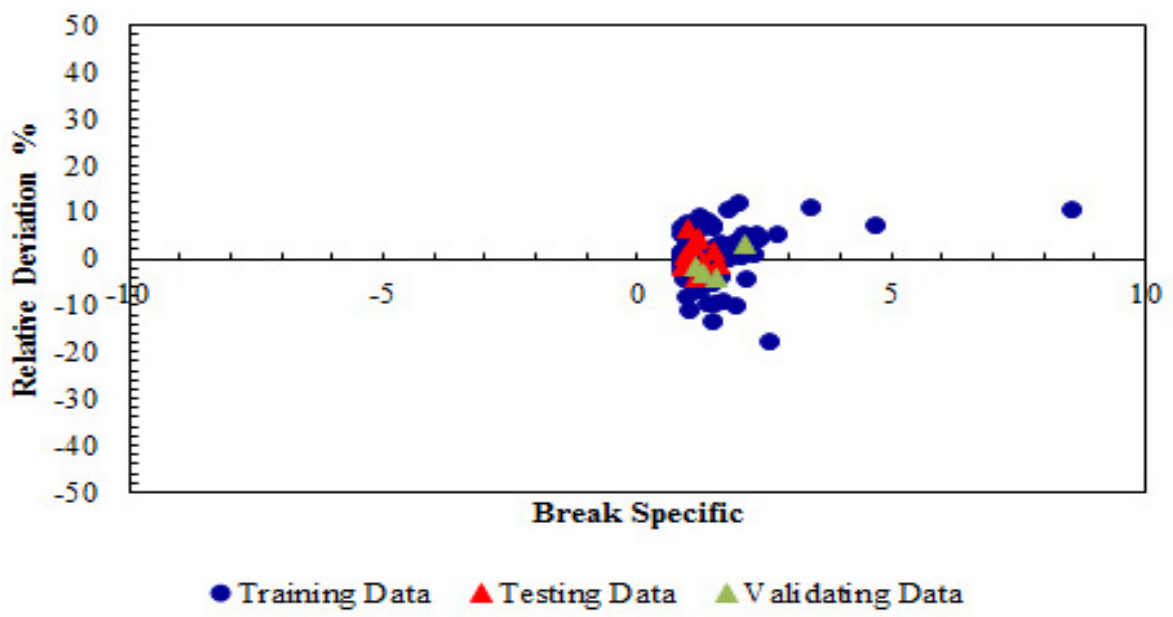

Fig. 2. Relative deviations between Brake Specific fuel consumption values estimated by LSSVM model and data measured in experimental condition $[46,47]$.

the majority of data points in the ranges $0 \leq H \leq H^{*}$ and $-3 \leq R \leq t 3$ divulges that both method evolvement and its estimations are done in applicability domain, which results in a statistically valid model. "Good High Leverage" points are placed in domain of $H^{*} \leq H$ and $-3 \leq R \leq 3$. The Good High Leverage can be opted as the ones, which are outside of applicability domain of the employed model. It means that the model cannot represent/predict the following data at all.

The points placed in the range of $R<-3$ or $3<R$ are opted as outliers of the model or "Bad High Leverage" points. These wrong estimations may be ascribed to the doubtful data.

\section{Results and discussions}

As mentioned earlier, two important parameters of the LSSVM model are $\gamma$ and $\sigma^{2}$. Based on the aforementioned procedure for determination of the addressed parameters
Table 1. The LSSVM model parameters.

\begin{tabular}{ccc}
\hline & $\gamma$ & $\sigma^{2}$ \\
\hline Brake specific fuel consumption & 3623.346 & 187.8732325 \\
Torque & 2037.8375 & 74.82365847 \\
Power & 634.38764 & 83.83649387 \\
\hline
\end{tabular}

in previous sections, the obtained values of the $\gamma$ and $\sigma^{2}$ for Brake Specific fuel consumption, Torque and power in the Stirling engine are reported in Table 1.

Based on the obtained values of $\gamma$ and $\sigma^{2}$ in proposed LSSVM model, scatter plot of the developed model outputs based on squared correlation coefficient $\left(R^{2}\right)$ is depicted in Figure 1. As illustrated in Figure 1, results of the LSSVM model fall on the diagonal line in lower boundary of the Brake Specific fuel consumption that means high precision of the developed model in the addressed boundary. However, few deviation from $Y=X$ line in intermediate and upper boundaries leads to higher uncertainty of the obtained results in the mentioned boundaries. To 


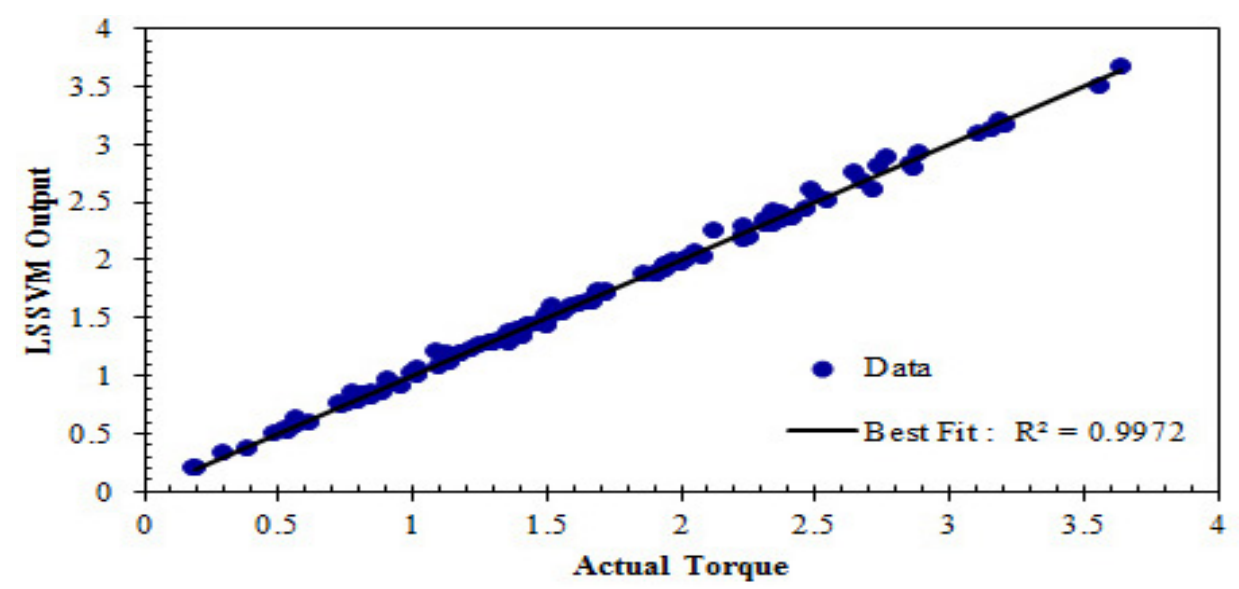

Fig. 3. Linear regression fit between the power estimated by LSSVM method and torque measured in experimental conditions $[46,47]$ for the studied systems.

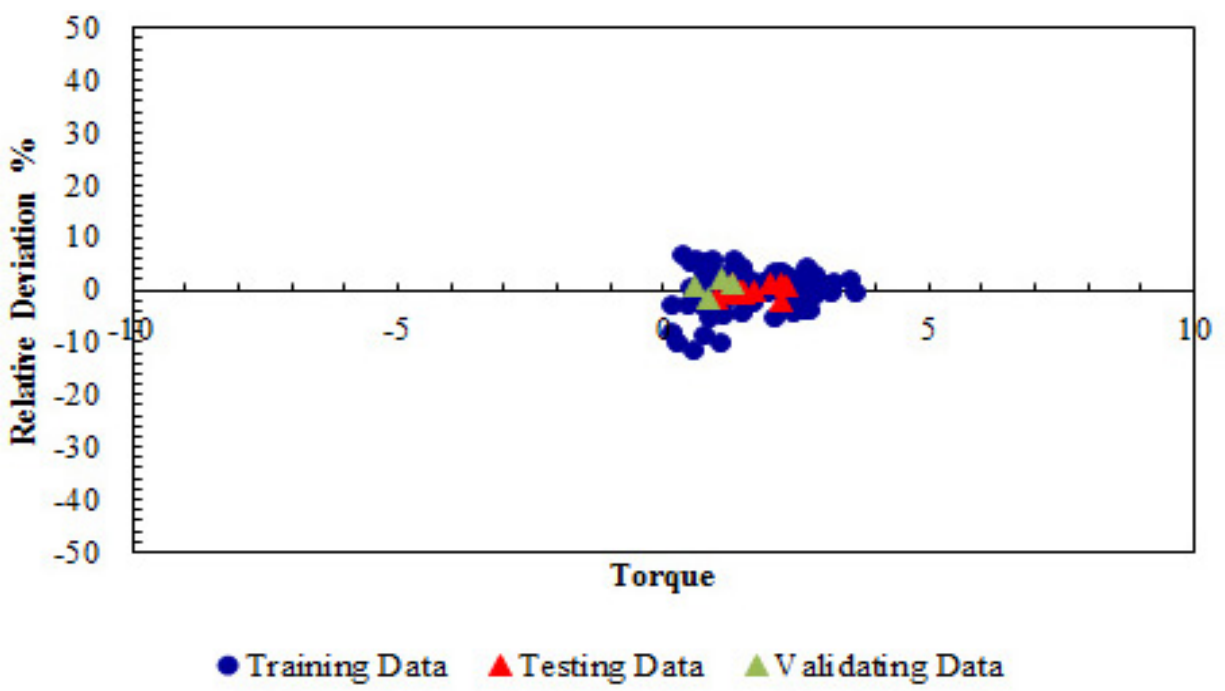

Fig. 4. Relative deviations between torque values estimated by LSSVM model and data measured in experimental condition $[46,47]$.

wrap up previous details in one smart plot, relative deviation of each LSSVM output against relevant measured Brake Specific fuel consumption values is illustrated in Figure 2.

As can be seen from Figure 2, the LSSVM model has acceptable deviation from corresponding actual values which is about $20 \%$, however three noisy points with high deviation from referred experimental value are observed. The calculated torque values in contrast with the experimental ones [46,47], are explained in Figure 3. As illustrated in Figure 3 the outputs gained by the LSSVM method for predicting torque have satisfactory agreement with experimental ones and the deviation is negligible for torque values between 0.5 and 4 . As demonstrated in Figure 4 , the errors for the LSSVM method for estimating the torque between 0.5 and $1.5 \mathrm{~N} . \mathrm{m}$ are intermediate and between 1.5 and 3.5 N.m are low. However two unacceptable points in lower boundary of the experimental torque values are observed. Due to the changing conditions in the laboratory, there is a possibility of error. The two data unacceptable are of these errors.

Figures 5 and 6 show the estimated values performing the LSSVM model developed in this research vs. measured values of the power for Stirling heat engine. Same as previous figures, Figure 5 illustrates the output results of suggested LSSVM model for Stirling engine power versus corresponding experimental power data. As previously mentioned falling on the diagonal line in regression plot leads to high precision of the implemented model. As can be seen from Figure 5, outcomes of LSSVM model between $100 \mathrm{~W}$ and $300 \mathrm{~W}$ have low deviation from experimental ones, although deviation from corresponding values between $300 \mathrm{~W}$ and $450 \mathrm{~W}$ is not negligible. Obviously the output results of LSSVM model in Figure 6 for $50 \mathrm{~W}$ through $450 \mathrm{~W}$ are collapsed around zero error line that means that error of the LSSVM method in this space is negligible. Despite the previous fact, two or three 
M.H. Ahmadi et al.: Mechanics \& Industry 17, 506 (2016)

Table 2. Criteria calculated for the LSSVM model proposed in this research.

\begin{tabular}{cccc}
\hline Statistical criteria & Brake specific fuel consumption & Torque & Power \\
\hline Training set & & & \\
$R^{2}$ & 0.9864 & 0.9971 & 0.9934 \\
Average absolute relative deviation (AARD) & 4.04 & 2.4293 & 2.7828 \\
Root mean square error (RMSE) & 0.0722 & 0.332 & 5.9655 \\
$N$ & 96 & 96 & 96 \\
Validation set & & & \\
$R^{2}$ & 0.9913 & 0.9963 & 0.995 \\
AARD & 2.0038 & 1.1289 & 1.3325 \\
RMSE & 0.211 & 0.228 & 3.4405 \\
$N$ & 5 & 5 & 5 \\
Test set & & & \\
$R^{2}$ & 0.9809 & 0.9989 & 0.9992 \\
AARD & 2.9537 & 0.9322 & 0.7966 \\
RMSE & 0.367 & 0.0113 & 1.2273 \\
$N$ & 11 & 11 & 11 \\
Total & & & \\
$R^{2}$ & 0.9862 & 0.9972 & 0.9939 \\
AARD & 3.8570 & 2.2376 & 2.5230 \\
RMSE & 0.06707 & 0.306 & 5.3874 \\
$N$ & 112 & 112 & 112 \\
\hline
\end{tabular}

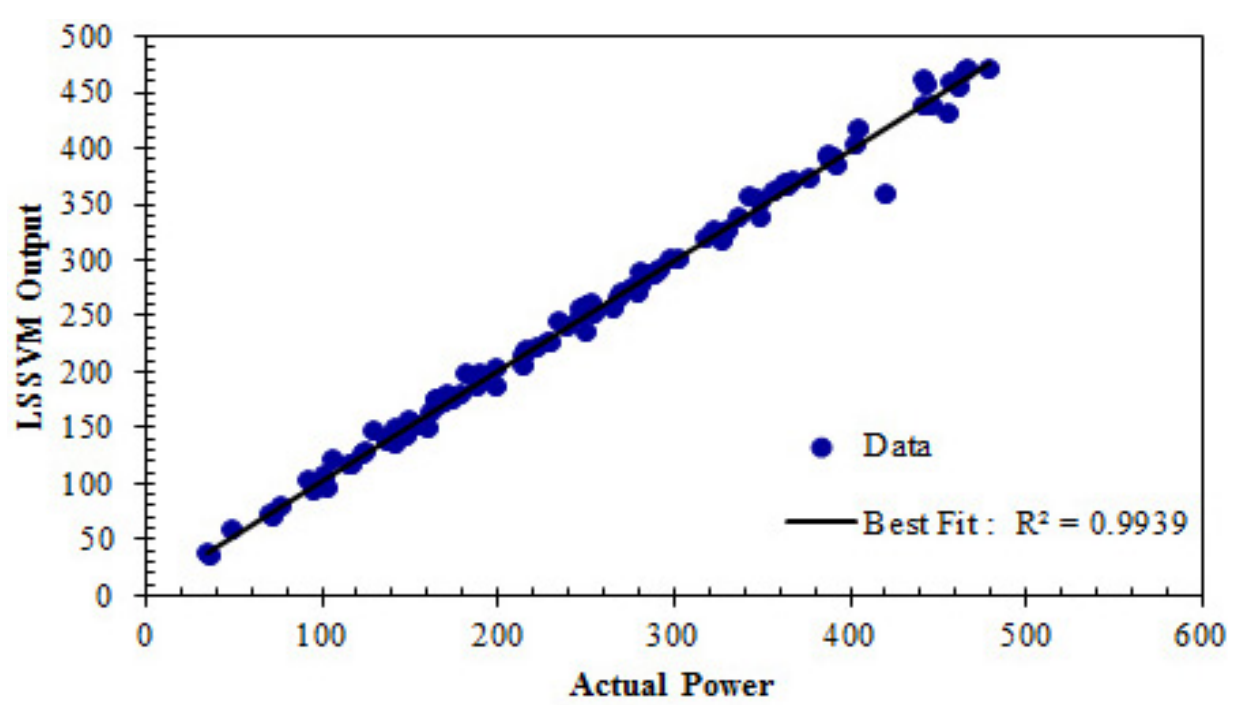

Fig. 5. Linear regression fit between the power estimated by LSSVM method and power measured in experimental conditions $[46,47]$ for the studied systems.

noisy points for power lower than $50 \mathrm{~W}$ are observed in the addressed figure.

To quantify all of the previous discussions, the statistical parameters of the proposed approach are summarized in Table 2 for training, validating and testing phases which include the $R^{2}$, AARD, and RMSE. As reported in Table 2, the developed LSSVM model has acceptable squared correlation coefficient in training, validating and testing phases for the three addressed targets being higher than 0.9. It is worth mentioning that approaching squared correlation coefficient value to one means that represented values by the LSSVM model cover the relevant actual value. Furthermore, average absolute relative deviation for the three mentioned outputs in training, validating and testing phases is less than $6 \%$ and it means the high accuracy of the suggested LSSVM model. Finally root mean square errors of the obtained results are in the adequate manner. The determination of the best learning algorithm and experimental data for Stirling engine are shown in Table 3.

Finally, Williams's plots of all the previous results are demonstrated in Figures 7 through 9. As depicted in Figure 7 , the warning Hat value of the brake specific values is 0.133929. Based on the obtained warning Hat value, the Hat value of any data samples greater than the addressed warning Hat value, means that the relevant output of the developed LSSVM model is not adequate and acceptable.

As shown in the Figure 7, one point is out of leverage based up the previous point and it means that these experimental data are doubtful. Moreover, the Williams 


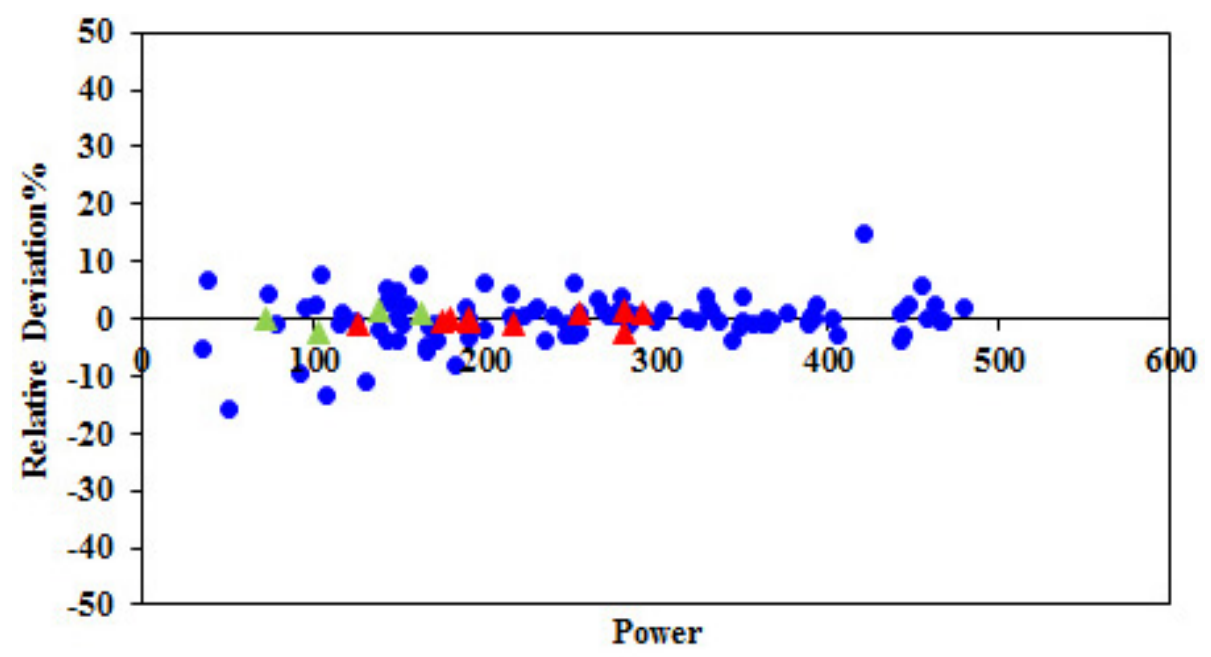

-Training Data $\Delta$ Testing Data $\Delta$ Validating Data

Fig. 6. Relative deviations between power values estimated by LSSVM model and data measured in experimental condition $[46,47]$.

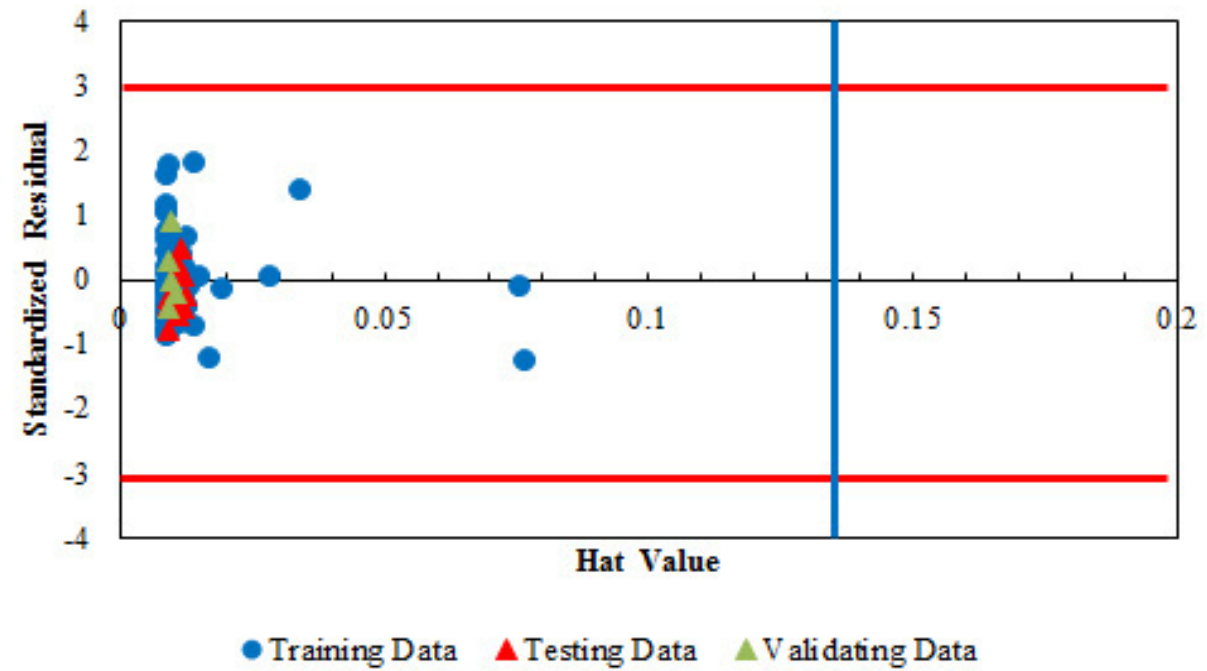

Fig. 7. Capability domain of the intelligent model proposed for estimating the Brake Specific fuel consumption of the Stirling engine and specifying of the possible uncertain real data. The $H^{*}$ value is 0.133929 .

plot of the LSSVM outcomes for the torque and the power are demonstrated in Figures 8 and 9. As can be seen in the aforementioned figures, coverage of the LSSVM model outputs in the ranges of $-3<R<+3,0<H<0.133929$ proves and confirms the robustness and low uncertainty of the proposed LSSVM model in prediction of Brake Specific fuel consumption, Torque and power of the Stirling engine. However, in the three depicted plots one point is observed in the outlier region.

\section{Conclusions}

Massive efforts were made for simplifying estimation of the Stirling engine parameters such as power, torque and Break specific fuel consumption. To assess the main end of this communication, a new type of SVM called
LSSVM was implemented to represent the addressed parameters of the Stirling engine. Moreover, statistical criteria such as AARD, RMSE and $R^{2}$ of the outputs of the developed LSSVM model determined and due to indicated statistical parameters, suggested LSSVM model outputs have low deviation from the corresponding actual Stirling engine data samples. Finally, Williams plots of the generated results were demonstrated in details. Based on the critical Hat value for the three addressed outputs of the Stirling engine, the proposed LSSVM model has satisfyingly performance. Obtained results from this paper could help to design more accurate Stirling engine system. We think that this application will be useful for other types of Stirling engines or other working conditions although the correct input data will increase the accuracy of the results. Therefore, studying the other free derivative optimization 


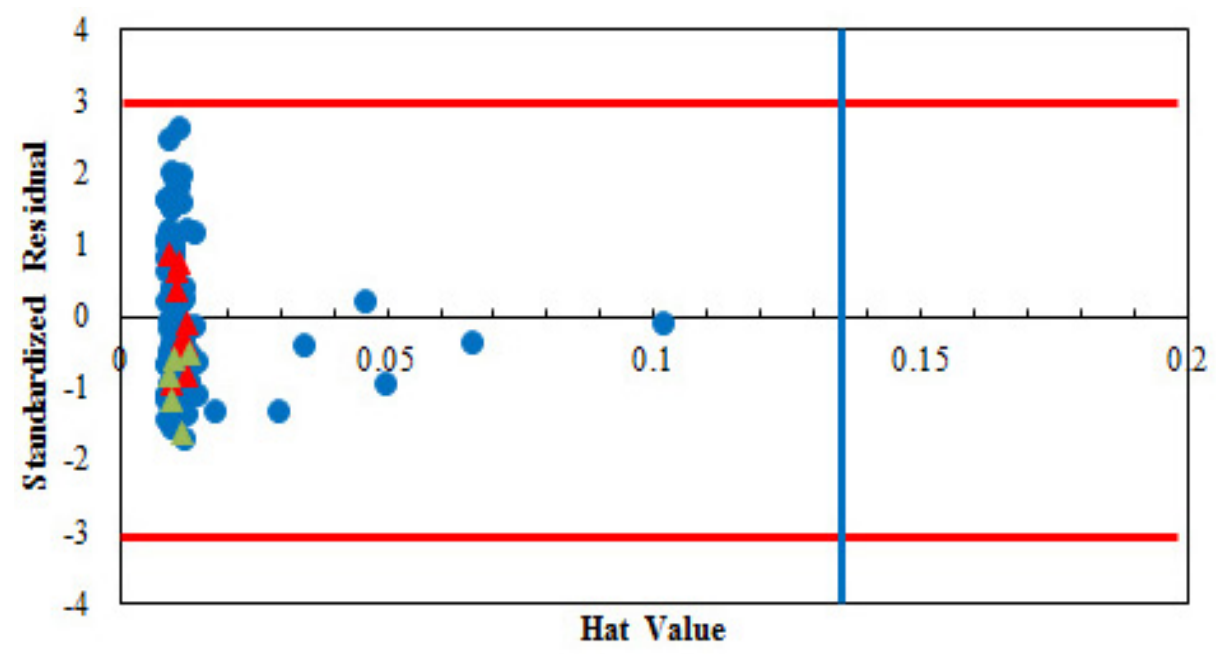

- Training Data $\Delta$ Testing Data $\Delta$ V alidating Data

Fig. 8. Capability domain of the intelligent model proposed for estimating the torque of the Stirling engine and specifying of the possible uncertain real data. The $H^{*}$ value is 0.133929 .

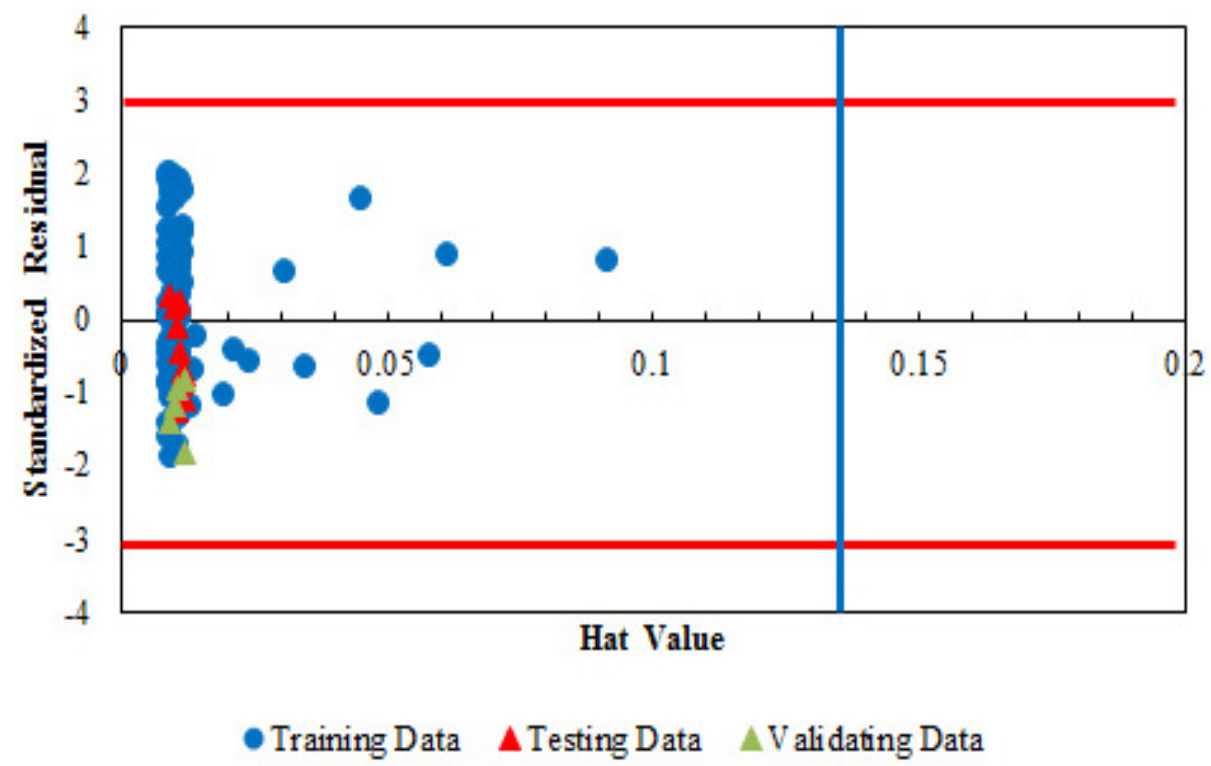

Fig. 9. Capability domain of the intelligent model proposed for estimating the Power of the Stirling engine and specifying of the possible uncertain real data. The $H^{*}$ value is 0.133929 .

methods on the models will be a part of our future investigations.

\section{Conflicts of Interest:}

The authors declare no conflict of interest.

\section{References}

[1] D.J. Shendage, S.B. Kedare, S.L. Bapat, An analysis of a beta type Stirling engine with rhombic drive mechanism, Renew. Energy 36 (2011) 289-297
[2] D.G. Thombare, S.K. Verma, Technological development in the Stirling cycle engines, Renew Sustain. Energy Rev. 12 (2008) $1-38$

[3] H. Karabulut, H.S. Yucesu, C. Cinar, Nodal analysis of a Stirling engine with concentric piston and displacer, Renew. Energy 31 (2006) 2188-2197

[4] H. Karabulut, H.S. Yucesu, C. Cinar, F. Aksoy, An experimental study on the development of a b-type Stirling engine for low and moderate temperature heat sources, Appl. Energy 86 (2009) 68-73

[5] E. Prodesser, Electricity production in rural villages with biomass Stirling engines, Renew. Energy 16 (1999) 10491052 
[6] A. Sripakagorn, C. Srikam, Design and performance of a moderate temperature difference Stirling engine, Renew. Energy 36 (2011) 1728-1733

[7] H. Karabulut, C. Cinar, E. Ozturk, H.S. Yucesu, Torque and power characteristics of a helium charged Stirling engine with a lever controlled displacer driving mechanism, Renew. Energy 35 (2010) 138-143

[8] C.H. Cheng, Y.J. Yu, Numerical model for predicting thermodynamic cycle and thermal efficiency of a beta-type Stirling engine with rhombic-drive mechanism, Renew. Energy 35 (2010) 2590-2601

[9] W.L. Chen, K.L. Wong, L.W. Po, A numerical analysis on the performance of a pressurized twin power piston gamma-type Stirling engine, Energy Convers. Manag. 62 (2012) 84-92

[10] F. Formosa, G. Despesse, Analytical model for Stirling cycle machine design, Energy Convers. Manag. 51 (2010) $1855-1863$

[11] R. Tol, Autoregressive conditional heteroscedasticity in daily wind speed measurements, Theor. Appl. Climatol. 56 (1997) 113-122

[12] J. Torres, A. Garcia, M.de Blas, A. de Francisco, Forecast of hourly average wind speed with ARMA models in Navarre (Spain), Sol. Energy 79 (2005) 65-77 Energies 5 (2012) 3346

[13] G.H. Riahy, M. Abedi, Short term wind speed forecasting for wind turbine applications using linear prediction method, Renew. Energy 33 (2008) 35-41

[14] Y.M. Atwa, E.F. El-Saadany, Annual wind speed estimation utilizing constrained grey predictor, IEEE Trans. Energy Conver. 24 (2009) 548-550

[15] T.H. El-Fouly, E.F. El-Saadany, M.M.A. Salama, Grey predictor for wind energy conversion systems output power prediction, IEEE Trans. Power Syst. 21 (2006) 1450-1452

[16] M. Lei, L. Shiyan, J. Chuanwen, L. Hongling, Z. Yan, A review on the forecasting of wind speed and generated power, Renew. Sustain. Energy Rev. 13 (2009) 915-920

[17] M.A. Mohandes, T.O. Halawani, S. Rehman, A.A. Hussain, Support vector machines for wind speed prediction, Renew. Energy 29 (2004) 939-947

[18] A. UlHaque, J.L. Meng, Short-term wind speed forecasting based on fuzzy artmap, Int. J. Green Energy 8 (2011) $65-80$

[19] Y.Y. Hong, H.L. Chang, C.S. Chiu, Hour-ahead wind power and speed forecasting using simultaneous perturbation stochastic approximation (SPSA) algorithm and neural network with fuzzy inputs, Energy 35 (2010) 38703876

[20] G.F. Fan, W.S. Wang, C. Liu, H.Z. Dai, Wind Power Prediction Based on Artificial Neural Network; Electric Power Research Institute, Beijing, China, 2008; pp. 118123

[21] A. Öztopal, Artificial neural network approach to spatial estimation of wind velocity data, Energy Convers. Manage. 47 (2006) 395-406

[22] T. Ackermann, Wind power in power systems, Wind Eng. 30 (2006) 447-449

[23] A. Kecebas, I. Yabanova, M. Yumurtacı, Artificial neural network modeling of geothermal district heating system thought exergy analysis, Energy Convers. Manag. 64 (2012) 206-212
[24] M. KianiDehKiani, B. Ghobadian, T. Tavakoli, A.M Nikbakht, G. Najafi, Application of artificial neural networks for the prediction of performance and exhaust emissions in SI engine using ethanol- gasoline blends, Energy 35 (2010) 65-69

[25] R.M. Balabin, E.I. Lomakina, R.Z. Safieva, Neural network (ANN) approach to biodiesel analysis: Analysis of biodiesel density, kinematic viscosity, methanol and water contents using near infrared (NIR) spectroscopy, Fuel 90 (2011) 2007-2015

[26] M.A. Ahmadi, M. Ebadi, P.S. Marghmaleki, M.M. Fouladi, Evolving Predictive Model to Determine Condensate-to-Gas Ratio in Retrograded Condensate Gas Reservoirs, Fuel C 12 (2014) 241-257

[27] M.A. Ahmadi, M. Ebadi, Evolving smart approach for determination dew point pressure through condensate gas reservoirs, Fuel B 117 (2014) 1074-1084

[28] S. Zendehboudi, M.A. Ahmadi, A. Bahadori, A. Shafiei, T. Babadagli, A Developed Smart Technique to Predict Minimum Miscible Pressure-EOR Implication, Canadian J. Chem. Eng. 91 (2013) 1325-1337

[29] A. Chouai, S. Laugier, D. Richon, Modeling of thermodynamic properties using neural networks: application to refrigerants, Fluid Phase Equilib. 199 (2002) 53-62

[30] L. Piazza, G. Scalabrin, P. Marchi, D. Richon, Enhancement of the extended corresponding states techniques for thermodynamic modelling, I. Pure fluids, Int. J. Refrig. 29 (2006) 1182-1194

[31] G. Scalabrin, P. Marchi, L. Bettio, D. Richon, Enhancement of the extended corresponding states techniques for thermodynamic modelling, II. Mixtures, Int. J. Refrig. 29 (2006) 1195-1207

[32] M.A. Ahmadi, Neural Network Based Unified Particle Swarm Optimization for Prediction of Asphaltene Precipitation, Fluid Phase Equilibria 314 (2012) 46-51

[33] M.H. Ahmadi, M.A. Ahmadi, M. Mehrpooya, M.A. Rosen, Using GMDH Neural Networks to Model the Power and Torque of a Stirling Engine, Sustainability 7 (2015) 2243-2255

[34] S. Toghyani, M.H. Ahmadi, A. Kasaeian, A.H. Mohammadi, Artificial neural network, ANN-PSO and ANN-ICA for modelling the Stirling engine, Int. J. Ambient Energy (2014) 1-13

[35] J.H. Holland, Adaptation in Natural and Artificial Systems, University of Michigan Press, Ann Arbor, 1975

[36] M. Hagan, H.B. Demuth, M.H. Beale, Neural Network Design, International Thomson Publishing, Boston, 2002

[37] J.A.K. Suykens, T. Van Gestel, J. De Brabanter, B. De Moor, J. Vandewalle, Least Squares Support Vector Machines, World Scientific, Singapore, 2002

[38] J.A.K. Suykens, J. Vandewalle, Least squares support vector machine classifiers, Neural Process. Lett. 9 (1999) 293-300

[39] K. Pelckmans, J.A.K. Suykens, T. Van Gestel, D. De Brabanter, L. Lukas, B. Hamers, B. De Moor, J. Vandewalle, LS-SVM lab: a Matlab/C Toolbox for Least Squares Support Vector Machines, Internal Report 02-44, ESATSISTA, K.U. Leuven, Leuven, Belgium, 2002

[40] M. Curilem, G. Acuña, F. Cubillos, E. Vyhmeister, Neural Networks and Support Vector Machine models applied to energy consumption optimization in semiautogeneous grinding, Chem. Eng. Trans. 25 (2011) 761-766 
[41] C. Cortes, V. Vapnik, Support-Vector Networks, Machine Learning 20 (1995), http://www.springerlink.com/ content/k238jx04hm87j80g/

[42] Support Vector Machine page, Wikipedia (accessed October 2011)

[43] R.M. Balabin, E.I. Lomakina, Support vector machine regression (LS-SVM) - an alternative to artificial neural networks (ANNs) for the analysis of quantum chemistry data? Phys. Chem. Chem. Phys. 13 (2011) 11710-11718

[44] R.M. Balabin, R.Z. Safieva, Near-infrared (NIR) spectroscopy for biodiesel analysis: fractional composition, iodine value, and cold filter plugging point from one vibrational spectrum, Energy Fuels 25 (2011) 2373-2382

[45] R.M. Balabin, S.V. Smirnov, Variable selection in nearinfrared spectroscopy: benchmarking of feature selection methods on biodiesel data, Anal. Chim. Acta 692 (2011) 63-72

[46] G.L. Ward, Performance characteristics of the Stirling engine. MSc thesis, University of Bath, 1972

[47] J.I Prieto, M.A. Gonzalez, C. Gonzalez, J. Fano, A new equation representing the performance of kinematic Stirling engines, Proc. Instn. Mech. Engrs. C 214 (2000) 449-464
[48] S.R. Gunn, M. Brown, K.M. Bossley, Network performance assessment for neuro fuzzy data modeling, Lect. Notes Comput. Sci. 1280 (1997) 313-323

[49] M.H. Ahmadi, M.A. Ahmadi, S.A. Sadatsakkak, M. Feidt, Connectionist intelligent model estimates output power and torque of Stirling engine, Renew. Sustain. Energy Rev. 50 (2015) 871-883

[50] H. Liu, X. Yao, R. Zhang, M. Liu, Z. Hu, B. Fan, Accurate quantitative structure-property relationship model to predict the solubility of C60 in various solvents based on a novel approach using a least-squares support vector machine, J. Phys. Chem. B 109 (2005) 20565-20571

[51] M.A. Ahmadi, M. Ebadi, S.M. Hosseini, Prediction break through time of water coning in the fractured reservoirs by implementing low parameter support vector machine approach, Fuel A 117 (2014) 579-589

[52] P.J. Rousseeuw, A.M. Leroy, Robust Regression and Outlier Detection, John Wiley \& Sons, New York, 1987

[53] C.R. Goodall, Computation Using the QR Decomposition, Handbook in Statistics, 9. Elsevier/North-Holland, Amsterdam, 1993

[54] P. Gramatica, Principles of QSAR models validation: internal and external, QSAR Comb. Sci. 26 (2007) 694-701 